This is a post-peer-review, pre-copyedit version of an article published in 'Journal of Youth and Adolescence'. The final authenticated version is available online at: https://doi.org/10.1007/s10964-015-0405-y.

\title{
Basic Psychological Need Satisfaction, Emotion Dysregulation, and Non-suicidal Self- Injury Engagement in Young Adults: An Application of Self-Determination Theory
}

\section{Abstract}

Non-suicidal self-injury (NSSI) is a public health concern that affects young adults at alarming rates. The present study examines the role of satisfaction of selfdetermination theory's three basic needs for autonomy, competence, and relatedness in young adults’ NSSI engagement. University students who reported ever having engaged in NSSI $\left(n=40,85 \%\right.$ female; $\left.M_{\text {age }}=20.10, S D=1.66\right)$ reported significantly lower levels of the satisfaction of all three needs, as well as more difficulties with all aspects of emotion regulation (non-acceptance of emotional responses, difficulty engaging in goal directed behavior, impulse control, lack of emotional awareness, limited access to regulation strategies, lack of emotional clarity), compared to students with no history of $\operatorname{NSSI}\left(n=46,91 \%\right.$ female; $\left.M_{\text {age }}=19.79, S D=1.37\right)$. Results of a logistic regression analysis revealed that need satisfaction added to the prediction of NSSI group membership after controlling for the effects of emotion regulation. Satisfaction of the need for competence and limited access to emotion regulation strategies accounted for significant variance in NSSI in the final model. The findings suggest that selfdetermination theory may be a useful framework under which to conceptualize NSSI and that the need for competence may be particularly salient for University students.

\section{Introduction}

Non-suicidal self-injury (NSSI) can be understood as the deliberate and selfinflicted destruction of body tissue without suicidal intent and for purposes that are not 
This is a post-peer-review, pre-copyedit version of an article published in 'Journal of Youth and Adolescence'. The final authenticated version is available online at: https://doi.org/10.1007/s10964-015-0405-y.

culturally sanctioned (American Psychiatric Association 2013). NSSI has been dubbed “common" in college populations (Whitlock et al. 2011) with results from a recent metaanalysis finding that, after controlling for methodological differences across studies, 13.4 $\%$ of young adults report ever having engaged in NSSI (Swannell et al. 2014).

Engagement in NSSI has important implications as prospective research has shown that individuals who engage in NSSI are at a sevenfold increased risk for later suicide attempt (Guan et al. 2012). Thus, NSSI constitutes a prevalent and important health issue, and developing a strong understanding of the mechanisms underlying this behavior is imperative to guide effective prevention and intervention efforts.

Although many functions and risk factors have been associated with NSSI engagement, difficulties with emotion regulation is one of the most empirically supported precursors to NSSI initiation (Andover and Morris 2014; Perez et al. 2012). Some debate exists around how to operationally define emotion regulation (see Gross 2015), however, within the NSSI literature, emotion regulation has most often been studied under Gratz and Roemer's (2004) conceptualization as:

Involving the (a) awareness and understanding of emotions, (b) acceptance of emotions, (c) ability to control impulsive behaviors and behave in accordance with desired goals when experiencing negative emotions, and (d) ability to use situationally appropriate emotion regulation strategies flexibly to modulate emotional responses as desired in order to meet individual goals and situational demands. (pp. 42-43)

Emotion dysregulation occurs when all or some of these abilities are impaired in an individual. 
This is a post-peer-review, pre-copyedit version of an article published in 'Journal of Youth and Adolescence'. The final authenticated version is available online at: https://doi.org/10.1007/s10964-015-0405-y.

Research applying this conceptualization of emotion regulation has consistently found associations between this construct and NSSI. The results of a MANOVA conducted with an undergraduate sample, revealed that individuals with a history of NSSI reported significantly more difficulties in all emotion regulation factors on Gratz and Roemer's (2004) Difficulties in Emotion Regulation Scale (DERS) except for lack of emotional awareness when compared to their non-self-injuring peers (Heath et al. 2008). In a sample of female undergraduate students, Gratz and Roemer (2008) found that the limited access to emotion regulation strategies subscale on the DERS as well as lack of emotional clarity, accounted for greater variance in NSSI above and beyond other subscales. Similarly, in a sample of adolescent inpatients, Perez et al. (2012) found that only limited access to emotion regulation strategies accounted for variance in NSSI when other aspects of emotion dysregulation, sex, and psychopathology were controlled for. Thus, research suggests that NSSI may be used as a strategy to address perceived deficits in emotion regulation skills (Andover and Morris 2014) and specifically targeting the thoughts and feelings that one has no way to regulate their overwhelming emotions may be important in clinical interventions.

Whereas emotion dysregulation constitutes a significant intrapersonal proximal risk factor for NSSI, other more distal environmental factors have been investigated as correlates of NSSI and may be viewed as stimuli events that elicit emotional arousal. Broadly, one such distal factor is the social context in which an individual dwells. The qualities of this context are likely to affect the individual's functioning. For example, does the context allow the individual to act volitionally, provide opportunities for success, and does it offer encouraging support from others? The present article applies 
This is a post-peer-review, pre-copyedit version of an article published in 'Journal of Youth and Adolescence'. The final authenticated version is available online at: https://doi.org/10.1007/s10964-015-0405-y.

self-determination theory (SDT) to guide our understanding of NSSI environmental correlates. Self-determination theory has furthered our understanding of who is at risk for suicidal ideation (Bureau et al. 2012), a phenomenon closely linked to NSSI, and many NSSI risk and protective factors are conceptually associated with the three basic needs proposed by SDT. Thus, it seems likely that self-determination theory may serve as a complementary framework to existent emotion regulation based models.

\section{Self-Determination Theory}

Self-determination theory is an organismic approach built on the assumption that people are actively involved in their own development with evolved tendencies towards growth and mastery (Ryan and Deci 2000b). The theory outlines three universal, innate needs that serve as the avenue through which the social context influences development throughout the lifespan: autonomy, competence, and relatedness. The need for autonomy is understood as our need for feeling that we are acting out of our own volition and in accordance with our personal values as opposed to feeling as though our behavior stems from coercion or pressure (Grolnick and Raftery-Helmer 2013). The need for competence reflects our inherent desire to feel effective when interacting with our environment (Deci and Ryan 2000). It follows that when our need for competence is fulfilled, feelings of self-efficacy and self-esteem may be at the center of more general feelings of well-being. The need for relatedness is our need for deep and meaningful connections with close others, as well as a need for broader connections to society in general. This need is satisfied when we experience social support and feel close to others (Deci and Ryan 2008). Self-determination theory posits that all three needs are essential; when they are fulfilled via the social context, an individual is in the position to maintain optimal 
This is a post-peer-review, pre-copyedit version of an article published in 'Journal of Youth and Adolescence'. The final authenticated version is available online at: https://doi.org/10.1007/s10964-015-0405-y.

functioning and achieve positive personal growth. However, when any one need is thwarted, an individual's overall well-being and psychological health are at risk (Ryan and Deci 2000a).

According to self-determination theory, an immediate cost of ill-being follows when the satisfaction of basic needs is obstructed. Furthermore, when needs are chronically left unfulfilled, individuals fall into maladaptive patterns where they may chase after empty extrinsic goals and/or use compensatory behaviors to temporarily relieve negative emotions aroused by need frustration. The effects of this need replacement and compensation result in further frustration and thus, perpetuate a cycle of non-optimal functioning (Vansteenkiste and Ryan 2013). Under an SDT perspective, non-suicidal self-injury would be considered a releasing self-control compensatory behavior resulting from unfulfilled basic psychological needs (Vansteenkiste and Ryan 2013). Research has supported the link between need satisfaction and self-control compensatory behaviors such as binge eating (Schuler and Kuster 2011) and smoking (Williams et al. 2009). However, whereas a self-determination theory perspective has been applied to understand the quality of motivation to undertake psychotherapeutic change engagement in patients with eating disorders who do and do not report NSSI (Vansteenkiste et al. 2013), need satisfaction has not yet been examined to understand this particular releasing self-control compensatory behavior. Although this has not yet been examined, many correlates of NSSI investigated in young adult populations are conceptually related to self-determination theory's three basic needs, further suggesting that SDT may be a useful theoretical framework under which to consider NSSI.

\section{Autonomy, Competence, and Relatedness Variables and NSSI in Young Adults}


This is a post-peer-review, pre-copyedit version of an article published in 'Journal of Youth and Adolescence'. The final authenticated version is available online at: https://doi.org/10.1007/s10964-015-0405-y.

Many NSSI correlates are conceptually related to self-determination theory's basic psychological needs of autonomy, competence, and relatedness. Although the need for autonomy has not been investigated as a risk factor for NSSI, research suggests that parental practices that undermine autonomy such as parental criticism, rigid family values, and parental control are associated with NSSI (e.g., Bureau et al. 2010; Hamza and Willoughby 2013; Yates et al. 2008). These variables do not assess participants' general autonomy support, instead, they are similar to autonomy support within the early caregiving environment, which Koestner et al. (1984) operationalize as: (a) providing rationale and explanation for behavioral requests; (b) recognizing the feelings and perspective of the child; (c) offering choices and encouraging initiative; and, (d) minimizing the use of controlling techniques. A limitation within this research is that most studies rely on young adults' retrospective memories of their childhood environments. The present study will address this limitation by asking participants about their current levels of satisfaction of autonomy in general.

Although low satisfaction of the need for competence has not been explored as a risk factor for NSSI, the conceptually related variables of self-esteem, negative selfappraisals, self-derogation, a sense of inadequacy about the self, feelings of worthlessness, and ineffectiveness are correlates of NSSI in young adults (e.g., Breen et al. 2013; Cawood and Huprich 2011; Rotolone and Martin 2012). In both qualitative and quantitative research, young adults who self-injure in university as well as those recruited through online communities, consistently report lower self-esteem and more feelings of inadequacy compared to their non-injuring peers. Furthermore, the written language of these young adults contains themes of self-derogation and worthlessness. It makes sense 
This is a post-peer-review, pre-copyedit version of an article published in 'Journal of Youth and Adolescence'. The final authenticated version is available online at: https://doi.org/10.1007/s10964-015-0405-y.

that these individuals would also report low levels of fulfillment of the need for competence, however, this self-determination theory construct has not yet been directly examined as a correlate in the NSSI literature.

In terms of self-determination theory's proposed need for relatedness, researchers and theoreticians suggest that engagement in NSSI may have a social function such that the act of NSSI may serve to strengthen affiliations and a sense of group belonging (e.g., Heath et al. 2009; Hilt and Hamm 2014; Nock 2009). This social function likely results from a lack of satisfaction of the need for relatedness where individuals engage in NSSI to feel connection and support from others. Indeed, aspects of relatedness such as social support in general, support from parents and friends, connectedness to parents, loneliness, and attachment have been explored in terms of their relation to the development and maintenance of NSSI in young adults, suggesting an influential relationship between the need for relatedness and NSSI (e.g., Bureau et al. 2010; Hallab and Covic 2010; Muehlenkamp et al. 2013; Taliaferro and Muehlenkamp 2015).

Despite the evidence suggesting a link between autonomy, competence, and relatedness associated variables and NSSI in young adults, self-determination theory's tenet that one potential consequence of blocking need satisfaction is the releasing selfcontrol compensatory behavior of NSSI, has yet to be directly empirically examined. Considering the empirical support showing the applicability of self-determination theory to other proposed releasing self-control compensatory behaviors (Schuler and Kuster 2011; Williams et al. 2009), it is likely that SDT could be a strong complementary tool to emotion regulation models that may serve to further our understanding of NSSI while taking into account the influence of the social environment. 
This is a post-peer-review, pre-copyedit version of an article published in 'Journal of Youth and Adolescence'. The final authenticated version is available online at: https://doi.org/10.1007/s10964-015-0405-y.

\section{Research Objectives}

The overall goal of the present study is to apply self-determination theory's basic psychological needs to understand and explain NSSI among a sample of young adults. Specifically, the first objective is to examine whether individuals who report having engaged in NSSI significantly differ from those with no history of NSSI on reported levels of satisfaction of the three needs (autonomy, competence, and relatedness) and on reported levels of the six emotion dysregulation factors (non-acceptance, goal, impulse, awareness, strategies, and clarity). Previous NSSI research with need related variables suggests that individuals with a history of NSSI should report lower satisfaction of the three needs compared to individuals with no history of NSSI (e.g., Hamza and Willoughby 2013; Taliaferro and Muehlenkamp 2015). Based on Gratz and Roemer's (2004) conceptualization of emotion regulation and previous research linking emotion dysregulation to NSSI, it was hypothesized that all six aspects of emotion dysregulation would significantly differ between groups, indicating that those with a history of NSSI would report increased difficulties with emotion regulation compared to individuals without a history of NSSI.

The second objective is to examine whether the basic need satisfaction of autonomy, competence, and relatedness add to the prediction of NSSI group membership over and above the well-established influence of difficulties in emotion regulation. Research with a similar sample (Gratz and Roemer 2008) suggests that limited access to emotion regulation strategies and lack of emotional clarity should account for greater variance in NSSI above and beyond other aspects of emotion regulation measured by the DERS. However, researchers have yet to examine the role of need satisfaction in NSSI 
This is a post-peer-review, pre-copyedit version of an article published in 'Journal of Youth and Adolescence'. The final authenticated version is available online at: https://doi.org/10.1007/s10964-015-0405-y.

engagement. Given the previously presented research suggesting variables related to these needs are indeed associated with NSSI engagement, it is hypothesized that the three basic needs will add to the prediction of NSSI group membership when the effects of emotion dysregulation are accounted for.

\section{Methods}

\section{Participants}

Participants were drawn from a large pre-existing dataset examining coping strategies among young adults from a large urban area Canadian university. The overall sample consisted of 1436 participants $(73.6 \%$ female, $26.3 \%$ male, and $.1 \%$ who did not report gender). Participants ranged in age from 17 to 42 years $\left(M_{\text {age }}=20.01\right.$ years, $S D$ $=2.30)$ and reported their place of birth as Canada (68 \%), United States (14 \%), East Asia (4\%), Europe (3\%), and Other (11\%). Exclusions based on age were of necessity as the current study focused on young adults; thus, only participants who reported ages ranging from 18 to 25 were included in the present study. A total of 32 participants were excluded based on this criterion, reducing the sample to 1404 participants. From the remaining sample, 114 participants $(8.12 \%)$ responded positively on a screening questionnaire that they had physically hurt themselves on purpose without suicidal intent at least once in their lives. Participants were invited to complete a follow up survey. Of the 114 participants who indicated NSSI engagement, 40 ( 34 female, 6 male; $M_{\mathrm{age}}=$ 20.10 years, $S D=1.66$ ) completed the measure of interest in a follow up survey and were included in the NSSI group. Participants in the NSSI group reported birthplace as follows: Canada (69 \%), United States (10\%), East Asia (6\%), Europe (2 \%), Other (13 $\%)$. 
This is a post-peer-review, pre-copyedit version of an article published in 'Journal of Youth and Adolescence'. The final authenticated version is available online at: https://doi.org/10.1007/s10964-015-0405-y.

Of the 40 participants, $30 \%$ reported engaging in NSSI within the last year, $27 \%$ within the last 2 years, $19 \%$ within the last three to 4 years, and $24 \%$ reported having engaged in the behavior more than 4 years ago. With regard to frequency, $12 \%$ reported having engaged in NSSI once, $20 \%$ reported two to four times, $29 \%$ reported five to ten times, $27 \%$ reported eleven to fifty times, $10 \%$ reported fifty-one to one-hundred times, and $2 \%$ reported having engaged in the behavior more than one-hundred times.

A control group of 46 participants (42 female, 4 male; $M_{\text {age }}=19.79, S D=1.37$ ) who had no history of NSSI and who completed the measure of interest in a follow up survey, was created from the overall sample. The NSSI group and control group did not significantly differ on gender, age, and country of birth.

\section{Measures}

\section{NSSI Screening Questionnaire}

The How I Deal with Stress Questionnaire (HIDS; Heath and Ross 2007) is a 29item self-report questionnaire developed to screen for self-injury. Each statement on the HIDS taps the frequency of use of both adaptive and maladaptive coping strategies on a four-point Likert scale ranging from 0 (Never) to 3 (Frequently). NSSI is embedded within these statements as a coping strategy ("physically hurt myself on purpose"). The HIDS also has a follow-up section in which participants are asked to provide additional information on NSSI and to indicate whether they had harmed themselves without suicidal intent to ensure reports of self-harm meet NSSI definition criteria. The HIDS has been used successfully in community settings to accurately screen for and identify youth who engage in NSSI (Ross and Heath 2002). For the present study, participants were 
This is a post-peer-review, pre-copyedit version of an article published in 'Journal of Youth and Adolescence'. The final authenticated version is available online at: https://doi.org/10.1007/s10964-015-0405-y.

included in the NSSI group if they indicated a 1 or above on the Likert scale for the NSSI item and completed the follow up section indicating that their self-injury was without suicidal intent.

\section{Need Fulfillment}

To examine the satisfaction of self-determination theory's three basic needs, the Basic Psychological Needs Scale-General Version (BPNS) was administered. This scale is adapted from the Basic Psychological Needs Satisfaction Scale-Work Version (Ilardi et al. 1993). The BPNS consists of 21 items that assess need satisfaction on three subscales: autonomy (7 items), competence (6 items), and relatedness ( 8 items). Participants are asked how true each statement is for them on a 7-point Likert Scale ranging from 1 (not at all true) to 7 (very true). An autonomy subscale item example is "I feel like I am free to decide for myself how to live my life," a competence subscale reverse-scored item example is "Often, I do not feel very competent," and a relatedness subscale item example is "People in my life care about me." Gagné (2003) reported construct validity with the three need subscales being positively related to maternal and paternal autonomy support. For the present study, reliability for each need subscale was as follows: autonomy $\alpha=.65$, competence $\alpha=.79$, and relatedness $\alpha=.83$. Due to poor internal consistency, item 20 "There is not much opportunity for me to decide how to do things" was removed from the autonomy subscale.

\section{Emotion Dysregulation}

The Difficulties in Emotion Regulation Scale (DERS; Gratz and Roemer 2004) is a 36-item self-report measure that assesses six components of emotion dysregulation 
This is a post-peer-review, pre-copyedit version of an article published in 'Journal of Youth and Adolescence'. The final authenticated version is available online at: https://doi.org/10.1007/s10964-015-0405-y.

based on Gratz and Roemer's (2004) model. The subscales are non-acceptance of emotional responses (non-acceptance; e.g., I pay attention to how I feel), difficulties engaging in goal directed behavior (goal; e.g., When I'm upset I have difficulty focusing on other things), impulse control difficulties (impulse; e.g., I experience my emotions as overwhelming and out of control), lack of emotional awareness (awareness; e.g., I pay attention to how I feel), limited access to emotion regulation strategies (strategies; e.g., When I'm upset, I believe there is nothing I can do to feel better), and lack of emotional clarity (clarity; e.g., I am clear about my feelings). Items are scored on a 5-point Likert scale ranging from 1 (almost never) to 5(almost always). The measure has demonstrated construct and predictive validity, and test-retest reliability across $4-8$ weeks $(p<.01$; Gratz and Roemer 2004). In the present study, each subscale had internal consistency that ranged from good to excellent (non-acceptance $\alpha=.93$, goal $\alpha=.90$, impulse $\alpha=.89$, awareness $\alpha=.85$, strategies $\alpha=.90$, clarity $\alpha=.84$ ).

\section{Procedure}

Data collection took place in undergraduate classes at a large urban university in Canada. Instructors from various courses and programs were contacted via email to explain the general purpose of the study and to request permission to present the study to their students during class time. During class visits, a research assistant introduced the study as an investigation of student stress and coping, using a scripted introduction while packages containing a consent form, a form to fill in their contact information if they agreed for further follow-up, and the HIDS were distributed. Students who were willing to participate were given 15 min to complete the package in class. Once completed, a 
This is a post-peer-review, pre-copyedit version of an article published in 'Journal of Youth and Adolescence'. The final authenticated version is available online at: https://doi.org/10.1007/s10964-015-0405-y.

research assistant collected the packages and provided participants with a debriefing sheet that gave contact information of the research team and mental health resources.

Participants who gave consent for follow up were emailed a battery of questionnaires, including the BPNS, that they could complete and email back at their convenience. Upon receipt of the completed follow-up questionnaires, the participants received another email providing the necessary debriefing information and received $\$ 25$ as well as a link to access resources should they require additional support. The data was then coded and entered into a database and no identifiable information was available through database access alone.

\section{Results}

Table 1 presents the means and standard deviations of the study variables by group. Table 2 presents the correlations of the study variables for the control group. Table 3 presents the correlations of the study variables for the NSSI group.

\section{Group Differences on Need Satisfaction and Emotion Dysregulation}

Prior to conducting analyses, all variables were examined through SPSS 22 for the accuracy of data entry, detect missing values, and fit between their distributions and assumptions of multivariate analyses. Questionnaires were considered to be invalid and participants were not included in analyses if more than $5 \%$ of their data was missing. If 5 $\%$ or less of the data was missing, missing values were estimated using the regression method in SPSS 22. To examine whether participants who have engaged in NSSI and participants with no history of NSSI differ on reported levels of self-determination theory's need satisfaction and reported levels of emotion dysregulation, two multivariate 
This is a post-peer-review, pre-copyedit version of an article published in 'Journal of Youth and Adolescence'. The final authenticated version is available online at: https://doi.org/10.1007/s10964-015-0405-y.

analyses of variance (MANOVAs) were conducted, one with satisfaction of the three needs as dependent variables, and one with the six emotion dysregulation subscales as dependent variables. Results of the first MANOVA revealed overall significant differences between groups on need satisfaction $F(3,82)=5.34, p=<.01$; Wilk's $\Lambda=$ .84 , partial $\eta^{2}=.16$. In support of our hypotheses, tests of between-subjects effects revealed that the NSSI group and control group significantly differed on their reported levels of satisfaction for all three needs. Specifically, participants in the NSSI group reported: significantly lower levels of autonomy satisfaction compared to participants in the control group, $F(1,84)=6.51, p<.05$, partial $\eta^{2}=.07$; significantly lower levels of competence satisfaction compared to participants in the control group, $F(1,84)=16.29$, $p<.001$, partial $\eta^{2}=.16$; and significantly lower levels of relatedness satisfaction compared to participants in the control group, $F(1,84)=6.56, p<.05$, partial $\eta^{2}=.07$. Overall power to detect the effects was excellent ranging from .713 to .979. Results of the second MANOVA revealed overall significant differences between groups on emotion dysregulation $F(6,79)=9.37, p=<.001$; Wilk's $\Lambda=.58$, partial $\eta^{2}=.42$. In support of our hypotheses, tests of between-subjects effects revealed that the NSSI group and control group significantly differed on all six emotion dysregulation subscales.

Specifically, participants in the NSSI group reported: significantly higher levels of nonacceptance of emotional responses compared to the control group, $F(1,84)=31.40$, $p<$ .001 , partial $\eta^{2}=.27$; significantly higher levels of difficulty engaging in goal-directed behavior compared to the control group $F(1,84)=17.82, p<.001$, partial $\eta^{2}=.18$; significantly higher levels of difficulties with impulse control compared to the control group $F(1,84)=24.85, p<.001$, partial $\eta^{2}=.23$; significantly higher levels of lack of 
This is a post-peer-review, pre-copyedit version of an article published in 'Journal of Youth and Adolescence'. The final authenticated version is available online at: https://doi.org/10.1007/s10964-015-0405-y.

emotional awareness compared to the control group $F(1,84)=6.94, p<.05$, partial $\eta 2=$ .08.; significantly higher levels of limited access to regulation strategies $F(1,84)=$ $46.17, p<.001$, partial $\eta^{2}=.36$; and significantly higher levels of lack of emotional clarity compared to the control group $F(1,84)=26.59, p<.001$, partial $\eta^{2}=.24$. Overall power to detect the effects was excellent ranging from .740 to 1.00 .

Need Satisfaction and Emotion Dysregulation as Predictors of NSSI Group Membership

To examine whether the satisfaction of self-determination theory's basic needs adds to the prediction of NSSI group membership after accounting for the effects of emotion dysregulation, a logistic regression analysis was conducted on NSSI group membership as the outcome.

The six emotion dysregulation predictors (non-acceptance, goal, impulse control, awareness, regulation strategies, clarity) were entered in Step 1 of the regression. The three need satisfaction predictors (autonomy, competence, and relatedness) were entered in Step 2 of the regression. A test of the first block model against a constant-only model was statistically significant, $\chi^{2}(6, N=86)=43.46, p<.001$ indicating that the emotion dysregulation predictors as a set reliably distinguished between those who have engaged in NSSI and those who have not. The model successfully predicted $80.4 \%$ of the individuals who had not engaged in NSSI and $72.5 \%$ of individuals who had engaged in NSSI, for an overall success rate of $76.7 \%$. In partial support of our hypothesis, according to the Wald criterion, only limited access to regulation strategies reliably predicted NSSI group membership, $\chi^{2}(1, N=86)=4.22, p<.05$. Addition of the need satisfaction variables in Step 2 significantly improved the model $\chi^{2}(3, N=86)=8.05, p<$ 
This is a post-peer-review, pre-copyedit version of an article published in 'Journal of Youth and Adolescence'. The final authenticated version is available online at: https://doi.org/10.1007/s10964-015-0405-y.

.05. Classification was improved with $84.8 \%$ of individuals who had no NSSI history successfully predicted, and $82.5 \%$ of those with a history of NSSI successfully predicted, for an overall success rate of $83.7 \%$. Table 4 shows regression coefficients, Wald statistics, odds ratios, and $95 \%$ confidence intervals for odds ratios for each of the nine predictors. According to the Wald criterion, limited access to regulation strategies remained a significant predictor of NSSI group membership, $\chi^{2}(1, N=86)=4.40, p<$ .05. Furthermore, in partial support of our hypothesis, the satisfaction of the need for competence $\chi^{2}(1, N=86)=6.20, p<.05$ significantly added to the prediction of NSSI group membership such that decreases in satisfaction of the need for competence significantly increased the odds of being classified in the NSSI group.

\section{Discussion}

Non-suicidal self-injury is a health concern in young adults that has been associated with suicide ideation and attempts (Guan et al. 2012; Paul et al. 2015). Many young adults who engage in NSSI describe themselves as having difficulties in emotion regulation, and this impairment constitutes a significant intrapersonal risk factor for NSSI (Andover and Morris 2014). Environmental risk factors have also been examined within the NSSI correlate literature and point towards the influence of the social context on NSSI behavior (e.g., Hamza and Willoughby 2013; Taliaferro and Muehlenkamp 2015). Although many of these risk factors are conceptually related to self-determination theory's three basic needs for autonomy, competence, and relatedness, this theory has not been applied to further our understanding of the underlying mechanisms behind documented associations. Thus, the present article applied self-determination theory to 
This is a post-peer-review, pre-copyedit version of an article published in 'Journal of Youth and Adolescence'. The final authenticated version is available online at: https://doi.org/10.1007/s10964-015-0405-y.

examine the relationship between need satisfaction, emotion dysregulation, and NSSI engagement among university students.

As hypothesized, participants who had engaged in NSSI reported significantly lower satisfaction of autonomy, competence, and relatedness, and reported significantly higher levels of non-acceptance of emotions, difficulties engaging in goal directed behavior when upset, difficulties with impulse control, difficulties being aware of their emotions, limited emotion regulation strategies, and difficulties with emotion clarity compared to participants with no NSSI history. The finding that individuals with a history of NSSI engagement reported greater emotion regulation difficulties is a link that has been well-established in the literature in both clinical and community samples (e.g., Gratz and Roemer 2008; Heath et al. 2008). The finding that those who engaged in NSSI reported significantly lower levels of satisfaction for autonomy, competence, and relatedness compared to individuals with no NSSI history gives preliminary support for the application of self-determination theory to further our understanding of NSSI.

To further assess the usefulness of SDT in explaining variability in NSSI engagement, the contribution of need satisfaction to the prediction of NSSI group membership was assessed after controlling for the six factors of emotion regulation. Results revealed that competence added to the prediction of NSSI engagement over and above the dimensions of emotion regulation, such that a decrease in reported satisfaction of the need for competence significantly increased young adults' odds of being classified in the NSSI group even when emotion regulation difficulties were accounted for. This finding highlighting the importance of competence, is in accordance with previous research showing that young adults who engage in NSSI have lower self-esteem and 
This is a post-peer-review, pre-copyedit version of an article published in 'Journal of Youth and Adolescence'. The final authenticated version is available online at: https://doi.org/10.1007/s10964-015-0405-y.

more feelings of worthlessness compared to young adults who do not engage in the behavior (Heath et al. 2009; Rotolone and Martin 2012), and with the self-derogation found in the written language of individuals sharing in NSSI online communities (Breen et al. 2013). It is likely that when faced with environments and experiences that are perceived as not providing positive and constructive feedback and that do not allow for opportunities that are optimally challenging, negative feelings towards the self may occur, and difficulties regulating these feelings lead to the engagement in NSSI. Under a self-determination theory perspective, this undermining of competence may become especially dangerous as continued need thwarting leads to lowered motivation or to motivation that is pressured or controlled. When individuals act from more controlled forms of motivation, they either fail to identify environments that provide need fulfillment or actively select environments that continue to thwart their needs, in turn leading to increased stress (Vansteenkiste and Ryan 2013; Weinstein and Ryan 2011).

The present study examined autonomy support in general, asking participants to what extent they felt that their environment allowed for opportunities to act volitionally and in accordance with personal values. The findings suggest that although the degree to which an individual's need for autonomy is supported may distinguish between those who engage and do not engage in NSSI, it may not be a useful predictor of NSSI group membership when the influence of difficulties in emotion regulation and satisfaction of competence are taken into account.

Similarly, contrary to expectations, satisfaction of the need for relatedness was not a significant predictor of NSSI engagement. While intrapersonal functions are most often endorsed by individuals who engage in NSSI (Klonsky 2007), self-injurers also 
This is a post-peer-review, pre-copyedit version of an article published in 'Journal of Youth and Adolescence'. The final authenticated version is available online at: https://doi.org/10.1007/s10964-015-0405-y.

endorse interpersonal reasons for the behavior (Nock 2009; Nock and Prinstein 2004).

Furthermore, young adults who engage in NSSI often report feelings of loneliness, and a lack of social support and connectedness to peers and family (e.g., Bureau et al. 2010; Muehlenkamp et al. 2013; Taliaferro and Muehlenkamp 2015; Whitlock et al. 2015). In the self-determination theory realm, the satisfaction of the need for relatedness is widely considered and studied as an important protective factor to stressful responses (Weinstein and Ryan 2011). Thus, it would seem likely that reported satisfaction of the need for relatedness would predict NSSI group membership. Similar to the satisfaction of autonomy, it could be that although the need for relatedness successfully discriminated between those who do and do not engage in NSSI, its explanatory power was not strong enough to predict NSSI group membership when entered along with emotion regulation variables and satisfaction of the need for competence. It could also be possible that this need may not be as salient a predictor of NSSI engagement because of an experienced sense of community among those who self-injure.

The act of NSSI may serve to strengthen affiliations and a sense of group belonging (Heath et al. 2009; Nock 2009). Indeed, recent research into NSSI ecommunities suggests that the Internet is a place where individuals who engage in NSSI connect with others to give and to elicit support (Lewis et al. 2012; Lewis and Michal 2014). Another possibility that relatedness did not significantly predict NSSI group membership in the present sample may be because the NSSI group included both participants who report not having engaged in the behavior for several years as well as those who have engaged in NSSI within the past year. Thus, there may be variability in need satisfaction within this group itself. It could be that participants who do not 
This is a post-peer-review, pre-copyedit version of an article published in 'Journal of Youth and Adolescence'. The final authenticated version is available online at: https://doi.org/10.1007/s10964-015-0405-y.

currently engage in NSSI have evolved greater autonomy and more supportive social environments, but that the thwarting effects of the need for competence is less amenable to change. Longitudinally examining need satisfaction differences between individuals who maintain versus stop NSSI would be important to understand if this is the case.

With regards to emotion regulation, our hypothesis was partially supported; while lack of emotional clarity was not a significant predictor of NSSI group membership, limited access to emotion regulation strategies retained significance even after the addition of the three needs variables. The importance of the emotion regulation dimension of limited access to emotion regulation strategies has been shown in previous research (Perez et al. 2012) and has been included in etiological models of NSSI (e.g., Chapman et al. 2006). Although in a similar sample, Gratz and Roemer (2008) found that a lack of emotional clarity, in addition to limited emotion regulation strategies, accounted for greater variance in NSSI above and beyond the other DERS subscales, clarity was not a significant predictor in the present sample. As with the role of satisfaction of the need for relatedness, whereas other aspects of emotion regulation were successful in distinguishing between those who do and do not engage in NSSI, only limited access to regulation strategies was a predictor of the behavior in this sample. Limited access to emotion regulation strategies has also been implicated in the cessation of NSSI. Whitlock et al. (2015) found that young adults who had a past history of frequent NSSI (>6 incidents) but who reported no longer engaging in the behavior reported more effective emotion regulation strategies as measured by the DERS subscale compared to young adults who reported currently engaging in the behavior. Furthermore, in qualitative analyses of past and current self-injurer's comments, an increase in emotion regulation 
This is a post-peer-review, pre-copyedit version of an article published in 'Journal of Youth and Adolescence'. The final authenticated version is available online at: https://doi.org/10.1007/s10964-015-0405-y.

skills was identified as the primary driver of NSSI cessation. Although the present study does not examine NSSI cessation, the finding that limited access to emotion regulation skills predicts NSSI engagement lends further support for the importance of this variable to NSSI and the idea that individuals who engage in NSSI feel as though they do not have adaptive means to successfully regulate their internal states.

It is likely that a young adults' feelings of having limited access to emotion regulation strategies may be linked to their feelings of satisfaction of the need for competence. Indeed, it makes sense that individuals who do not feel that their need for competence is being supported would also feel incompetent when asked to find alternative healthy strategies to self-regulate. It could be that when the need for competence is chronically left unfulfilled, emotion regulation is negatively affected, and that this translates into the use of NSSI to modulate emotions. A similar pathway is put forth under self-determination theory where a lack of parental autonomy support leads children to regulate their emotions in maladaptive ways which results in internalizing and/or externalizing problems (Ryan et al. 2006).

There are several limitations to the present study. Although the study drew from a large dataset on stress and coping, the lifetime prevalence rate of NSSI was lower than what has previously been reported in young adult community samples (e.g., Serras et al. 2010; Whitlock et al. 2015). NSSI prevalence rates have been found to range widely within the literature and to vary depending on NSSI definition and how an NSSI screening question is presented, with higher prevalence reported when a checklist of NSSI behaviors is presented compared to one Likert scale question (Swannell et al. 2014). Although it is likely that this lower prevalence rate is due to the Likert 
This is a post-peer-review, pre-copyedit version of an article published in 'Journal of Youth and Adolescence'. The final authenticated version is available online at: https://doi.org/10.1007/s10964-015-0405-y.

methodology, it may be possible that the present sample represents an atypical group of university students and that the findings may not be generalizable to other young adults. Furthermore, this lower prevalence rate resulted in a small sample size. Future research should look beyond convenience sampling methods to increase sample size and power.

Second, although the majority of the participants within the NSSI group indicated NSSI engagement within the last year or 2 years, participants who indicated NSSI engagement more than two years ago were also included in the group. Recent research has shown differences between individuals who currently engage in NSSI and those who have a history of the behavior but who do not report current engagement (e.g., Taliaferro and Muehlenkamp 2015; Whitlock et al. 2015). Thus, by placing all individuals with a history of NSSI in the NSSI group, potential between group differences may have been overlooked. Furthermore, because a portion of individuals within the NSSI group reported not currently engaging in NSSI, it is likely that they may have made gains in their emotion regulation strategies, potentially weakening this construct in the final analysis. A larger sample size would be important in order to have adequate power to determine differences between multiple categories of self-injurer status.

Third, less than $50 \%$ of the participants who originally completed the screening questionnaire agreed to follow up, making selection bias a possible limitation. Considering that only a third of the final participants in the NSSI group indicated engaging in NSSI within the last year and that the majority of participants reported engaging in NSSI ten times or less, it could be that those who chose to continue in the study were more likely to be individuals with less severe NSSI and who felt that they had recovered from NSSI. If so, this selection bias could have translated into less striking 
This is a post-peer-review, pre-copyedit version of an article published in 'Journal of Youth and Adolescence'. The final authenticated version is available online at: https://doi.org/10.1007/s10964-015-0405-y.

differences on need satisfaction and emotion regulation between the NSSI and control groups. However, because NSSI is cyclical in nature, with individuals relapsing even 2 years after having stopped the behavior (Walsh 2006) and because NSSI is a risk factor for more severe self-injury and suicide attempts (Guan et al. 2012; Nock et al. 2006), an understanding of mild NSSI in self-selected samples is important.

Fourth, while low levels of need satisfaction have been measured and associated with maladaptive outcomes and psychological distress (e.g., Costa et al. 2015; Przybylski et al. 2014), the self-determination theory literature has begun moving beyond this approach to include measures of need frustration to predict maladaptive outcomes (Vansteenkiste and Ryan 2013). Future research examining non-suicidal self-injury would benefit from incorporating a measure of need frustration.

Finally, the cross-sectional nature of the research impedes any conclusions for directionality or causality. It seems likely that self-determination theory may be a complementary framework to emotion regulation models in that it helps to explain external stimuli that precede overwhelming emotions. This direction may intuitively make sense and be supported by theory, however, the present findings included individuals who had not engaged in NSSI for more than 2 years, perhaps indicating that NSSI engagement may have a lasting impact on later need fulfillment. Longitudinal prospective research is needed to evaluate the theoretically proposed timelines and to examine the direction of these relationships.

\section{Conclusion}


This is a post-peer-review, pre-copyedit version of an article published in 'Journal of Youth and Adolescence'. The final authenticated version is available online at: https://doi.org/10.1007/s10964-015-0405-y.

The current findings suggest that self-determination theory may be complementary to emotion regulation models to understand, study, and treat NSSI in young adults. Working within an SDT framework provides direction for clinical interventions and prevention programs. We know that the social context plays a crucial role in supporting an individual's potential versus stimulating their vulnerabilities (Ryan and Deci 2000a), thus, it would be important to target the environments that young adults find themselves into maximize the opportunities to behave autonomously, to experience competence, and to feel supported. Similarly, young adults' faulty perceptions of their environments should be addressed and reframed in order to help them to view contexts as more supportive. Results from the present study specifically suggest that targeting young adults' emotion regulation strategies and need for competence may be important in treating NSSI. For example, clinicians could help clients to see the adaptive means that they already use to regulate emotions and can help clients to increase this toolset. When giving clients homework, clinicians should ensure that the tasks are optimally challenging, that is, difficult, yet achievable, thus, supporting the young adults' need for competence. Indeed, results from a meta analysis of over 180 different data sets confirm that approaches to health care that support the patients' autonomy lead to increases in satisfaction of autonomy, competence, and relatedness and ultimately predict moderate to strong levels of patient well-fare in physical and mental health ( $\mathrm{Ng}$ et al. 2012). In addition to lending prevention and intervention avenues, self-determination theory provides a well-established theoretical framework with which to organize and understand previous findings on NSSI risk and protective factors in young adults. In sum, although further replication and future investigation is needed, it appears that need satisfaction 
This is a post-peer-review, pre-copyedit version of an article published in 'Journal of Youth and Adolescence'. The final authenticated version is available online at: https://doi.org/10.1007/s10964-015-0405-y.

may contribute to our understanding of NSSI even after the well-established factor of emotion dysregulation is accounted for.

\section{References}

American Psychiatric Association. (2013). Diagnostic and statistical manual of mental disorders (5th ed.). Washington, DC: Author. 
This is a post-peer-review, pre-copyedit version of an article published in 'Journal of Youth and Adolescence'. The final authenticated version is available online at: https://doi.org/10.1007/s10964-015-0405-y.

Andover, M. S., \& Morris, B. W. (2014). Expanding and clarifying the role of emotion regulation in nonsuicidal self-injury. Canadian Journal of Psychiatry, 59(11), 569-575. Retrieved from http://www-scopuscom.proxy3.library.mcgill.ca/inward/record.url?eid=2-s2.084911926203\&partnerID=40\&md5=be8da5cc1d505e59abde0953deacf69d.

Breen, A. V., Lewis, S. P., \& Sutherland, O. (2013). Brief report: Non-suicidal self-injury in the context of self and identity development. Journal of Adult Development, 20, 57-62. doi:10.1007/s10804-013-9156-8.

Bureau, J. S., Mageau, G. A., Vallerand, R. J., Rousseau, F. L., \& Otis, J. (2012). Selfdetermination: A buffer against suicide ideation. Suicide and Life-Threatening Behavior, 42(4), 377-393. doi:10.1111/j.1943-278X.2012.00097.x.

Bureau, J.-F., Martin, J., Freynet, N., Poirier, A., Lafontaine, M.-F., \& Cloutier, P. (2010). Perceived dimensions of parenting and non-suicidal self-injury in young adults. Journal of Youth and Adolescence, 39(5), 484-494. doi:10.1007/s10964-009-9470-4.

Cawood, C. D., \& Huprich, S. K. (2011). Late adolescent nonsuicidal self-injury: The roles of coping style, self-esteem, and personality pathology. Journal of Personality Disorders, 25(6), 765-781. doi:10.1521/pedi.2011.25.6.765.

Chapman, A. L., Gratz, K. L., \& Brown, M. Z. (2006). Solving the puzzle of deliberate selfharm: The experiential avoidance model. Behavior Research and Therapy, 44(3), 371394. doi:10.1016/j.brat.2005.03.005.

Costa, S., Soenens, B., Gugliandolo, M., Cuzzocrea, F., \& Larcan, R. (2015). The mediating role of experiences of need satisfaction in associations between parental psychological control 
This is a post-peer-review, pre-copyedit version of an article published in 'Journal of Youth and Adolescence'. The final authenticated version is available online at: https://doi.org/10.1007/s10964-015-0405-y.

and internalizing problems: A study among Italian college students. Journal of Child and Family Studies, 24(4), 1106-1116. doi:10.1007/s10826-014-9919-2.

Deci, E. L., \& Ryan, R. M. (2000). The "what" and "why" of goal pursuits: Human needs and the self-determination of behavior. Psychological Inquiry, 11(4), 227-268. doi:10.1207/S15327965PLI1104_01.

Deci, E. L., \& Ryan, R. M. (2008). Self-determination theory: A macrotheory of human motivation, development, and health. Canadian Psychology/Psychologie canadienne, 49(3), 182-185. doi:10.1037/a0012801.

Gagné, M. (2003). The role of autonomy support and autonomy orientation in prosocial behavior engagement. Motivation and Emotion, 27(3), 199-223. doi:10.1023/A:1025007614869.

Gratz, K. L., \& Roemer, L. (2004). Multidimensional assessment of emotion regulation and dysregulation: Development, factor structure, and initial validation of the difficulties in emotion regulation scale. Journal of Psychopathology and Behavioral Assessment, 26, 41-54. doi:10.1023/B:JOBA.0000007455.08539.94.

Gratz, K. L., \& Roemer, L. (2008). The relationship between emotion dysregulation and deliberate self-harm among female undergraduate students at an urban Commuter University. Cognitive Behavior Therapy, 37, 14-25. doi:10.1080/16506070701819524.

Grolnick, W. S., \& Raftery-Helmer, J. N. (2013). The importance of autonomy for development and well-being. In B. W. Sokol, F. M. E. Grouzet, \& U. Muller (Eds.), Self-regulation and autonomy: Social and developmental dimensions of human conduct (pp. 141-164). New York, NY: Cambridge Press. 
This is a post-peer-review, pre-copyedit version of an article published in 'Journal of Youth and Adolescence'. The final authenticated version is available online at: https://doi.org/10.1007/s10964-015-0405-y.

Gross, J. J. (2015). Emotion regulation: Current status and future prospects. Psychological Inquiry, 26, 1-26. doi:10.1080/1047840X.2014.940781.

Guan, K., Fox, K. R., \& Prinstein, M. J. (2012). Non-suicidal self-injury as a time-invariant predictor of adolescent suicidal ideation and attempts in a diverse community sample. Journal of Consulting and Clinical Psychology, 80(2), 842-849. doi:10.1037/a0029429.

Hallab, L., \& Covic, T. (2010). Deliberate self-harm: The interplay between attachment and stress. Behavior Change, 27(2), 93-103. doi:10.1375/bech.27.2.93.

Hamza, C. A., \& Willoughby, T. (2013). Nonsuicidal self-injury and suicidal behavior: A latent class analysis among young adults. PLoS ONE, 8(3), e59955. doi:10.1371/journal.pone.0059955.

Heath, N. L., \& Ross, S. (2007). How i deal with stress. McGill University, Montreal, Quebec, Canada (unpublished instrument).

Heath, N. L., Ross, S., Toste, J. R., Charlebois, A., \& Nedecheva, T. (2009). Retrospective analysis of social factors and nonsuicidal self-injury among young adults. Canadian Journal of Behavioral Science/Revue canadienne des sciences du comportement, 41(3), 180-186. doi:10.1037/a0015732.

Heath, N. L., Toste, J. R., Nedecheva, T., \& Charlebois, A. (2008). An examination of nonsuicidal self-injury among college students. Journal of Mental Health Counseling, 30(2), 137-156. Retrieved from 
This is a post-peer-review, pre-copyedit version of an article published in 'Journal of Youth and Adolescence'. The final authenticated version is available online at: https://doi.org/10.1007/s10964-015-0405-y.

http://amhca.metapress.com.proxy3.library.mcgill.ca/app/home/main.asp?referrer=defaul t.

Hilt, L., \& Hamm, E. (2014). Peer influences on non-suicidal self-injury and disordered eating. In L. Claes \& J. J. Muehlenkamp (Eds.), Non-suicidal self-injury in eating disorders (pp. 255-272). Berlin: Springer.

Ilardi, B. C., Leone, D., Kasser, T., \& Ryan, R. M. (1993). Employee and supervisor ratings of motivation: Main effects and discrepancies associated with job satisfaction and adjustment in a factory setting. Journal of Applied Social Psychology, 23(21), 1789_1805. doi:10.1111/j.1559-1816.1993.tb01066.x.

Klonsky, E. D. (2007). The functions of deliberate self-injury: A review of the evidence. Clinical Psychology Review, 27(2), 226-239. doi:10.1016/j.cpr.2006.08.002.

Koestner, R., Ryan, R. M., Bernieri, F., \& Holt, K. (1984). Setting limits on children's behavior: The differential effects of controlling vs. informational styles on intrinsic motivation and creativity. Journal of Personality, 52(3), 233-248. doi:10.1111/j.14676494.1984.tb00879.x.

Lewis, S. P., Heath, N. L., Michal, N. J., \& Duggan, J. M. (2012). Non-suicidal self-injury, youth, and the Internet: What mental health professionals need to know. Child and Adolescent Psychiatry and Mental Health, 6(13), 1-9. doi:10.1186/1753-2000-6-13.

Lewis, S. P., \& Michal, N. J. (2014). Start, stop, and continue: Preliminary insight into the appeal of self-injury e-communities. Journal of Health Psychology,. doi: $10.1177 / 1359105314527140$. 
This is a post-peer-review, pre-copyedit version of an article published in 'Journal of Youth and Adolescence'. The final authenticated version is available online at: https://doi.org/10.1007/s10964-015-0405-y.

Muehlenkamp, J., Brausch, A., Quigley, K., \& Whitlock, J. (2013). Interpersonal features and functions of nonsuicidal self-injury. Suicide and Life-Threatening Behavior, 43, 67-80. doi:10.1111/j.1943-278X.2012.00128.x.

Ng, J. Y. Y., Ntoumanis, N., Thorgenson-Ntoumani, C., Deci, E. L., Ryan, R. M., Duda, J. L., \& Williams, G. C. (2012). Self-determination theory applied to health contexts: A metaanalysis. Perspectives on Psychological Science, 7(4), 325-340. doi: $10.1177 / 1745691612447309$.

Nock, M. K. (2009). Why do people hurt themselves? New insights into the nature and functions of self-injury. Current Directions in Psychological Science, 18(2), 78-83. doi:10.1111/j.1467-8721.2009.01613.x.

Nock, M. K., Joiner, T. E, Jr, Gordon, K. H., Lloyd-Richardson, E., \& Prinstein, M. J. (2006). Non-suicidal self-injury among adolescents: Diagnostic correlates and relation to suicide attempts. Psychiatry Research, 144, 65-72. doi:10.1016/j.psychres.2006.05.010.

Nock, M. K., \& Prinstein, M. J. (2004). A functional approach to the assessment of selfmutilative behavior. Journal of Consulting and Clinical Psychology, 72(5), 885-890. doi:10.1037/0022-006X.72.5.885.

Paul, E., Tsypes, A., Eidlitz, L., Ernhout, C., \& Whitlock, J. (2015). Frequency and functions of non-suicidal self-injury: Associations with suicidal thoughts and behaviors. Psychiatry Research, 225(3), 276-282. doi:10.1016/j.psychres.2014.12.026.

Perez, J., Venta, A., Garnaat, S., \& Sharp, C. (2012). The difficulties in Emotion Regulation Scale: Factor structure and association with nonsuicidal self-injury in adolescent 
This is a post-peer-review, pre-copyedit version of an article published in 'Journal of Youth and Adolescence'. The final authenticated version is available online at: https://doi.org/10.1007/s10964-015-0405-y.

inpatients. Journal of Psychopathology and Behavioral Assessment, 34(3), 393-404. doi:10.1007/s10862-012-9292-7.

Przybylski, A. K., Deci, E. L., Rigby, C. S., \& Ryan, R. M. (2014). Competence-impeding electronic games and players' aggressive feelings, thoughts, and behaviors. Journal of Personality and Social Psychology, 106(3), 441-457. doi:10.1037/a0034820.

Ross, S., \& Heath, N. (2002). A study of the frequency of self-mutilation in a community sample of adolescents. Journal of Youth and Adolescence, 31, 67-77. doi:10.1023/A:1014089117419.

Rotolone, C., \& Martin, G. (2012). Giving up self-injury: A comparison of everyday social and personal resources in past versus current self-injurers. Archives of Suicide Research, 16(2), 147-158. doi:10.1080/13811118.2012.667333.

Ryan, R. M., \& Deci, E. L. (2000a). The darker and brighter sides of human existence: Basic psychological needs as a unifying concept. Psychological Inquiry, 11(4), 319-338. doi:10.1207/S15327965PLI1104_03.

Ryan, R. M., \& Deci, E. L. (2000b). Self-determination theory and the facilitation of intrinsic motivation, social development, and well-being. American Psychologist, 55, 68-78. doi:10.1037/0003-066X.55.1.68.

Ryan, R. M., Deci, E. L., Grolnick, W. S., \& La Guardia, J. G. (2006). The significance of autonomy and autonomy support in psychological development and psychopathology. In D. Cicchetti \& D. Cohen (Eds.), Developmental psychopathology: Vol. 1. Theory and methods (2nd ed., pp. 295-849). New York: Wiley. 
This is a post-peer-review, pre-copyedit version of an article published in 'Journal of Youth and Adolescence'. The final authenticated version is available online at: https://doi.org/10.1007/s10964-015-0405-y.

Schuler, J., \& Kuster, M. (2011). Binge eating as a consequence of unfulfilled basic needs: The moderating role of implicit achievement motivation. Motivation and Emotion, 35, 89-97. doi:10.1007/s11031-010-9200.

Serras, A., Saules, K. K., Cranford, J. A., \& Eisenberg, D. (2010). Self-injury, substance use, and associated risk factors in a multi-campus probability sample of college students. Psychology of Addictive Behaviors, 24, 119-128. doi:10.1037/a0017210.

Swannell, S. V., Martin, G. E., Page, A., Hasking, P., \& St John, N. J. (2014). Prevalence of nonsuicidal self-injury in nonclinical samples: Systematic review, meta-analysis and meta-regression. Suicide and Life-Threatening Behavior, 44(3), 273-303. doi:10.1111/sltb.12070.

Taliaferro, L. A., \& Muehlenkamp, J. J. (2015). Factors associated with current versus lifetime self-injury among high school and college students. Suicide and Life-Threatening Behavior, 45, 84-97. doi:10.1111/sltb.12117.

Vansteenkiste, M., Claes, L., Soenens, B., \& Verstuyf, J. (2013). Motivational dynamics among eating disordered patients with and without non-suicidal self-injury: A self-determination theory approach. European Eating Disorders Review, 21, 209-214. doi:10.1002/erv.2215.

Vansteenkiste, M., \& Ryan, R. M. (2013). On psychological growth and vulnerability: Basic psychological need satisfaction and need frustration as a unifying principle. Journal of Psychotherapy Integration, 23(3), 263-280. doi:10.1037/a0032359.

Walsh, B. W. (2006). Treating self-injury: A practical guide. New York, NY: Guilford Press. 
This is a post-peer-review, pre-copyedit version of an article published in 'Journal of Youth and Adolescence'. The final authenticated version is available online at: https://doi.org/10.1007/s10964-015-0405-y.

Weinstein, N., \& Ryan, R. M. (2011). A self-determination theory approach to understanding stress incursion and responses. Stress and Health, 27, 4-17. doi:10.1002/smi.1368.

Whitlock, J., Muehlenkamp, J., Purington, A., Eckenrode, J., Barreira, P., Baral Abrams, G., et al. (2011). Nonsuicidal self-injury in a college population: General trends and sex differences. Journal of American College Health, 59(8), 691-698.

doi:10.1080/07448481.2010.529626.

Whitlock, J., Prussien, K., \& Pietrusza, C. (2015). Predictors of self-injury cessation and subsequent psychological growth: Results of a probability sample survey of students in eight universities and colleges. Child and Adolescent Psychiatry and Mental Health, 9, 112. doi:10.1186/s13034-015-0048-5.

Williams, G. C., Patrick, H., Niemiec, C. P., Ryan, R. M., \& Deci, E. L. (2009). The importance of supporting autonomy and perceived competence in facilitating long-term tobacco abstinence. Annals of Behavioral Medicine, 37, 315-324. doi:10.1007/s12160-009-9090y.

Yates, T. M., Tracy, A. J., \& Luthar, S. S. (2008). Nonsuicidal self-injury among "privileged" youths: Longitudinal and cross-sectional approaches to developmental process. Journal of Consulting and Clinical Psychology, 76, 52-62. doi:10.1037/0022-006X.76.1.52. 
This is a post-peer-review, pre-copyedit version of an article published in 'Journal of Youth and Adolescence'. The final authenticated version is available online at: https://doi.org/10.1007/s10964-015-0405-y.

\section{Tables and Figures}

\begin{tabular}{|l|l|l|}
\hline \multicolumn{2}{|l|}{ Table 1 Means and standard deviations of DERS and CINSS subscales for control and NSSI } \\
\hline Vroups & Control (n = 46) & NSSI (n $=40)$ \\
\hline & M (SD) & M (SD) \\
\hline Non-acceptance & $10.89(3.78)$ & $16.78(5.86)$ \\
\hline Goal & $13.74(4.43)$ & $17.80(4.47)$ \\
\hline Impulse & $9.85(3.58)$ & $14.60(5.21)$ \\
\hline Awareness & $12.89(4.80)$ & $15.58(4.61)$ \\
\hline Strategies & $15.02(4.56)$ & $23.35(6.72)$ \\
\hline Clarity & $10.65(2.55)$ & $14.05(3.54)$ \\
\hline Autonomy & $30.85(5.14)$ & $28.00(5.18)$ \\
\hline Competence & $31.65(5.77)$ & $26.50(6.06)$ \\
\hline Relatedness & $45.04(7.73)$ & $40.78(7.68)$ \\
\hline
\end{tabular}


This is a post-peer-review, pre-copyedit version of an article published in 'Journal of Youth and Adolescence'. The final authenticated version is available online at: https://doi.org/10.1007/s10964-015-0405-y.

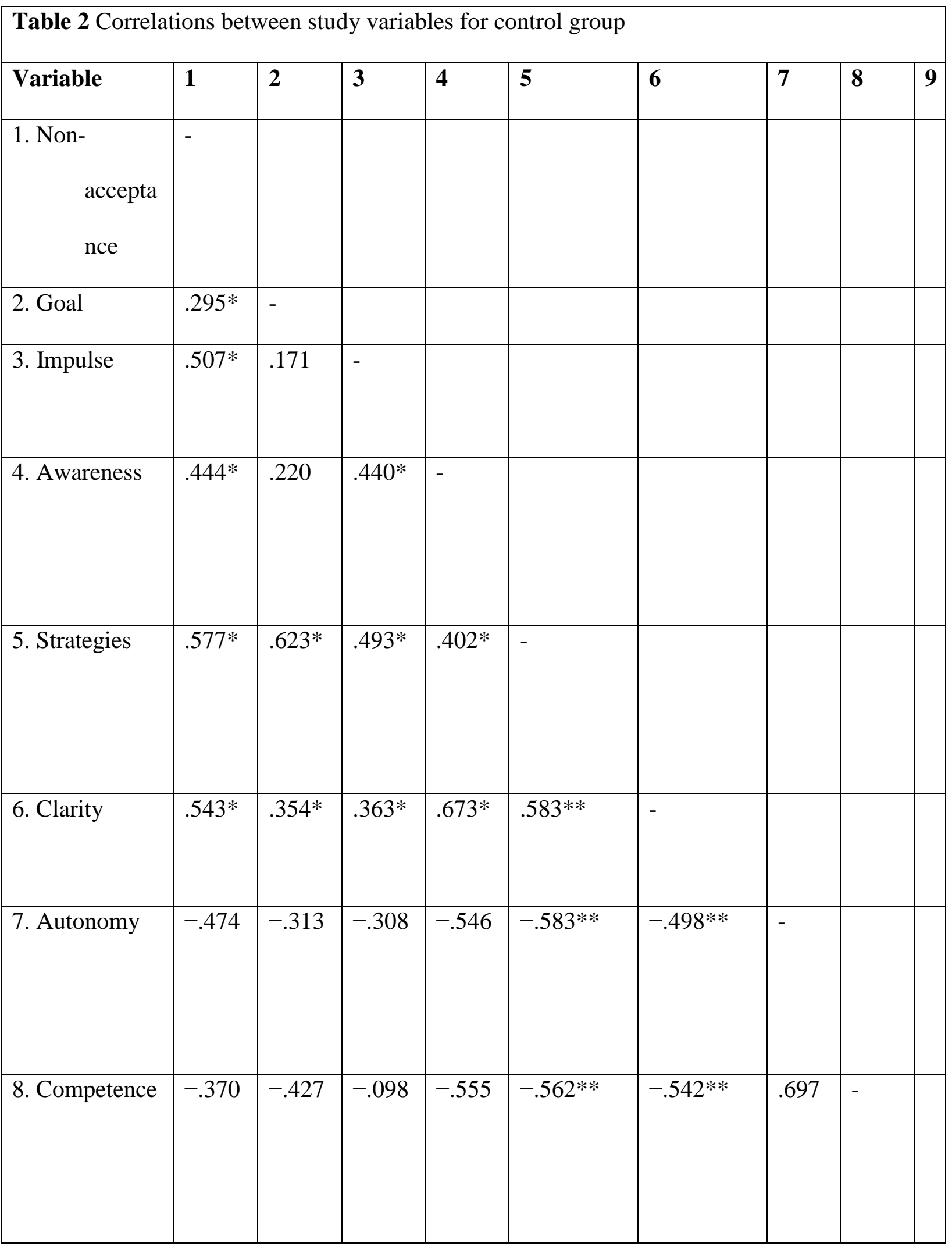


This is a post-peer-review, pre-copyedit version of an article published in 'Journal of Youth and Adolescence'. The final authenticated version is available online at: https://doi.org/10.1007/s10964-015-0405-y.

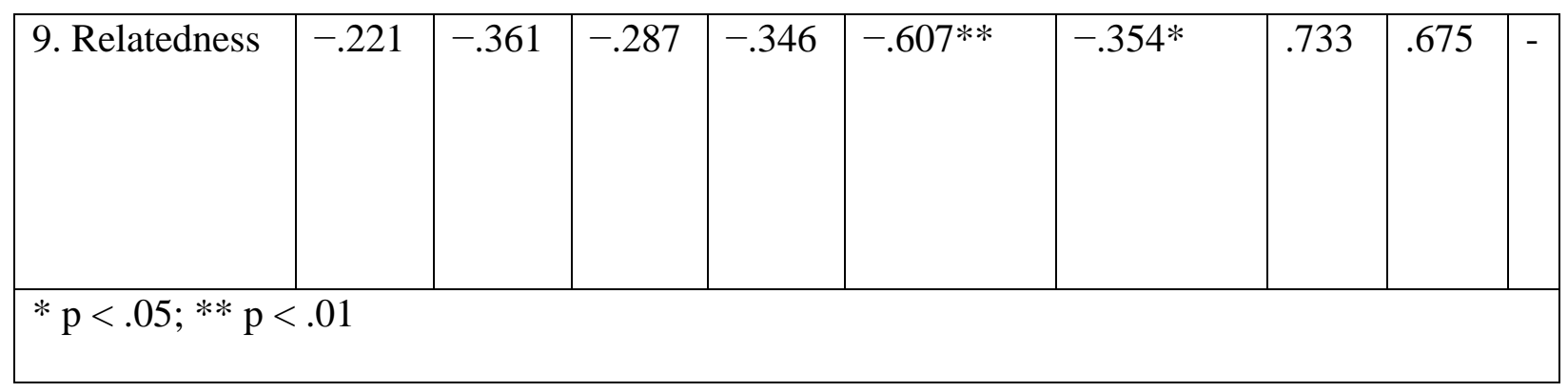

\begin{tabular}{|c|c|c|c|c|c|c|c|c|c|}
\hline Variable & 1 & 2 & 3 & 4 & 5 & 6 & 7 & 8 & 9 \\
\hline 1. Non-acceptance & - & & & & & & & & \\
\hline 2. Goal & $.354 *$ & - & & & & & & & \\
\hline 3. Impulse & $.361 *$ & $.419 * *$ & - & & & & & & \\
\hline 4. Awareness & .132 & .038 & .228 & - & & & & & \\
\hline 5. Strategies & $.375^{*}$ & $.588 * *$ & $.747 * *$ & .182 & - & & & & \\
\hline 6. Clarity & $.410^{*}$ & $.359^{*}$ & .234 & $.420 *$ & $.306^{*}$ & - & & & \\
\hline 7. Autonomy & -.240 & -.179 & $-.350^{*}$ & -.167 & -.381 & -.03 & - & & \\
\hline 8. Competence & -.170 & -.130 & -.085 & -.183 & -.120 & -.16 & $.668^{*}$ & - & \\
\hline 9. Relatedness & .030 & -.171 & -.041 & -.289 & -.141 & -.23 & $.476^{*}$ & $.527 *$ & - \\
\hline
\end{tabular}


This is a post-peer-review, pre-copyedit version of an article published in 'Journal of Youth and Adolescence'. The final authenticated version is available online at: https://doi.org/10.1007/s10964-015-0405-y.

\begin{tabular}{|c|c|c|c|c|c|}
\hline dysregula & $n$ and 1 & atisfaction variab & & & \\
\hline \multirow[t]{2}{*}{ Variables } & \multirow[t]{2}{*}{$\mathbf{B}$} & \multirow{2}{*}{$\begin{array}{l}\text { Wald Chi } \\
\text { square }\end{array}$} & \multirow[t]{2}{*}{$\operatorname{Exp}(B)^{a}$} & \multicolumn{2}{|c|}{$95 \% \mathrm{CI}^{\mathrm{b}}$} \\
\hline & & & & Lower & Upper \\
\hline \multicolumn{6}{|l|}{ Step 1} \\
\hline Non-acceptance & .08 & .98 & 1.08 & .92 & 1.27 \\
\hline Goal & -.01 & .02 & .99 & .85 & 1.15 \\
\hline Impulse & .03 & .10 & 1.03 & .86 & 1.23 \\
\hline Awareness & -.06 & .62 & .94 & .81 & 1.10 \\
\hline Strategies & .18 & $4.22 *$ & 1.19 & 1.01 & 1.41 \\
\hline Clarity & .23 & 2.67 & 1.26 & .96 & 1.65 \\
\hline \multicolumn{6}{|l|}{ Step 2} \\
\hline Autonomy & .23 & 4.10 & 1.25 & 1.00 & 1.56 \\
\hline Competence & -.25 & $6.20 *$ & .78 & .64 & .95 \\
\hline Relatedness & .03 & .16 & 1.03 & .90 & 1.17 \\
\hline \multicolumn{6}{|c|}{$\begin{array}{l}\text { Strategies retained significance in Step 2. Change in }-2 \text { Log Likelihood }=67.290, \mathrm{p}<.05 \\
{ }^{\mathrm{a}} \operatorname{Exp}(\mathrm{B})=\text { Odds ratio for each predictor }\end{array}$} \\
\hline \multicolumn{6}{|c|}{$\begin{array}{l}{ }^{\mathrm{b}} \text { Confidence interval is created around } \operatorname{Exp}(\mathrm{B}) \text {, statistically significant if } 1 \text { is not in the interval } \\
* \mathrm{p}<.05\end{array}$} \\
\hline
\end{tabular}

